\title{
Efeitos do intervalo pós-conhecimento de resultados na aquisição do arremesso da Bocha
}

\author{
Márcio M. Vieira $1,3,4$ \\ Fernando C. M. Ennes 1,4 \\ Guilherme M. Lage 1,2,5 \\ Leandro R. Palhares ${ }^{1}$ \\ Herbert Ugrinowitsch ${ }^{1}$ \\ Rodolfo N. Benda ${ }^{1}$
}

https://doi.org/10.5628/rpcd.06.01.50

\author{
${ }^{1}$ Universidade Federal de Minas Gerais \\ Escola de Educação Física, Fisioterapia e Terapia \\ Ocupacional, Grupo de Estudos em Desenvolvimento e \\ Aprendizagem Motora, Belo Horizonte, Brasil \\ ${ }^{2}$ Universidade Fumec, Brasil \\ ${ }^{3}$ Centro Universitário de Belo Horizonte, Brasil \\ ${ }^{4}$ Universidade Estácio de Sá, Brasil \\ ${ }^{5}$ Faculdades Unidas do Norte de Minas Gerais, Brasil
}

RESUMO

Com o objetivo de verificar os efeitos do intervalo após o fornecimento do conhecimento de resultados na aquisição do arremesso da bocha, trinta e seis universitários foram separados, formando três grupos: sem intervalo pós-conhecimento de resultados, intervalo de 5 segundos de pós-conhecimento de resultados e intervalo de 10 segundos de pós-conhecimento de resultados. A tarefa consistiu em arremessar uma bola em direção a um alvo circular posicionado no chão, com o objetivo de atingir um círculo no centro do alvo, que recebia a pontuação 6 . A cada $10 \mathrm{~cm}$ do círculo central, uma nova área foi delimitada e decrescia em um ponto recebendo a área mais externa a pontuação 1 . O experimento foi dividido em fase de aquisição (120 tentativas a 6,5 m de distância) e testes de Transferência 1 (10 tentativas sem conhecimento de resultados), Transferência 2 e 3 (10 tentativas cada sem conhecimento de resultados a 7,5 m). Não foram observadas diferenças estatísticas na análise da média do escore e do coeficiente de variação, não caracterizando efeito do intervalo pós-CR sobre a aprendizagem de habilidades motoras na condição utilizada.

Palavras-chave: aprendizagem motora, feedback, conhecimento de resultados.

\section{ABSTRACT}

Effects of Results' Post-Knowledge Interval in the Acquisition of the Bocce Throw

Aiming to verify the post-knowledge of results interval effects in the acquisition of the bocce throw, thirty-six college students of both sexes were separated in three groups: no post- knowledge of results interval, 5 -seconds post- knowledge of results interval and 10-seconds postknowledge of results interval. The task consisted of throwing a ball towards a circular target positioned in the floor aiming to reach a circle in the center of the target, which received score 6 . A new area was demarcated at each $10 \mathrm{~cm}$ of the central circle and decreased in one point receiving score 1 in the more external area. The experiment was divided in acquisition phase (120 trials at $6.5 \mathrm{~m}$ distance) and transfer test 1 (10 trials without knowledge of results at the same distance), transfer tests 2 and 3 (10 trials each one without knowledge of results at $7.5 \mathrm{~m}$ distance). Statistical differences were not observed in mean scores and coefficient of variation, that didn't determine the effect of the post-knowledge of results interval on learning motor skills in the condition used.

Key Words: motor learning, feedback, knowledge of results. 


\section{INTRODUÇÃO}

A manipulação da informação do conhecimento de resultado (CR), quanto ao aspecto temporal, produz três intervalos: pré-conhecimento de resultado, intervalo entre o término da tarefa e a apresentação do conhecimento de resultados (12); pós-conhecimento de resultado, intervalo de tempo entre a apresentação do conhecimento de resultados e a próxima tentativa (10); e o intervalo inter-tentativas, que separa uma resposta da tentativa seguinte (10). A dificuldade de se isolar os intervalos do conhecimento de resultados tem produzido evidências contraditórias quanto ao efeito das variáveis que se relacionam ao seu tempo de apresentação $(9,13,14)$. Por exemplo, quando se mantém constante o intervalo pré-conhecimento de resultado e se manipula o intervalo pós-conhecimento de resultado tem-se duas variáveis de influência (9), pois se altera o intervalo inter-tentativas. Essa característica do tempo de apresentação do conhecimento de resultado constitui uma limitação metodológica.

Bilodeau e Bilodeau (3) sugeriram que intervalos inter-tentativas mais longos ocasionavam decréscimo na aprendizagem. No entanto, estudos com utilização de testes de transferência em seus desenhos experimentais mostraram que o aumento do intervalo inter-tentativas ocasionou uma melhoria na performance (9). Ao se estudar o intervalo intertentativas, verificou-se que o intervalo pós-conhecimento de resultado é a única variável a interferir no desempenho (2).

O intervalo pré-conhecimento de resultados tem o papel de fortalecer o feedback intrínseco (informação recebida de fontes internas como a audição, propriocepção, visão e outros), pois oferece ao indivíduo tempo para analisar a tentativa executada (12). No intervalo pós-conhecimento de resultado é concedido ao sujeito um período de tempo para que o conhecimento de resultado seja processado, a fim de facilitar a performance subseqüente (4). Nesse intervalo, são comparados o feedback intrínseco e o conhecimento de resultado. No geral, os resultados têm mostrado que o intervalo pós-conhecimento de resultado é determinante para aquisição de habilidades $(8,9,15)$, podendo afetar a performance de forma mais efetiva que o intervalo pré-conhecimento de resultado. A duração do intervalo pós-conheci- mento de resultado depende da complexidade da tarefa, da natureza do feedback e da questão da demanda de cognição $(9,2)$. Sobre a demanda de cognição, se os intervalos forem curtos não proporcionam tempo suficiente para analisar o conhecimento de resultado e planejar a nova tentativa, e se longos podem causar o esquecimento dos pontoschave da habilidade (15), o que sugere um ponto intermediário na extensão desses intervalos. Os intervalos estudados variam desde 1 segundo (15) até 1 hora (3), sendo que 5, 6 e 10 segundos são aqueles que apresentam melhores desempenhos $(5,6,8,15)$. Apesar das crianças precisarem de maiores intervalos que adultos para alcançar a mesma performance, Gallagher e Thomas (4) sugeriram que não fosse maior que 12 segundos.

Todavia, outros fatores podem interferir nesse intervalo, o que impede que seu tempo seja estabelecido com precisão.

Em sua maioria, os estudos sobre o intervalo pósconhecimento de resultado apresentaram apenas fase de aquisição, e indicaram que intervalos mais longos, maiores que 5 segundos, produziram melhores efeitos sobre a performance $(3,8,15)$, quando comparados a intervalos mais curtos (13, 14). Segundo Travlos e Pratt (14), o número de estudos com teste de retenção em seu desenho experimental é reduzido e, desses, apenas Magill (6) encontrou diferenças nos efeitos da variação dos intervalos entre curtos (5 segundos) e longos (20 segundos), o que dificulta uma análise mais profunda do efeito do intervalo pós-conhecimento de resultado. Some-se a essa inconsistência nos resultados a própria dificuldade em assumir um intervalo de tempo preciso, passível de generalização sem uma devida consideração às características da tarefa e da natureza do feedback (7).

Apesar de Magill (7) apresentar evidências que o feedback aumentado, informação recebida de fontes externas (9), pode ser suprimido, mesmo se tratando de tarefas presentes em situações com maior complexidade que as tarefas de laboratório, Becker (1) ressalta que o uso de tarefas de maior complexidade parece ser o componente necessário para evidenciar a real influência do efeito da variação do intervalo pós-conhecimento de resultados sobre a aquisição de habilidades motoras. 
Assim, este estudo tem como objetivo verificar a influência do intervalo pós-conhecimento de resultado na aquisição de uma tarefa complexa em situação real de ensino-aprendizagem.

\section{MATERIAIS E MÉTODO}

Participaram do estudo 36 universitários voluntários de ambos os sexos, com idade média de 24,83 \pm 3,94 anos. Utilizou-se como tarefa o arremesso da bocha, um jogo de origem européia que utiliza uma bola de material sintético e maciço com peso entre 1400 e 1700 gramas e diâmetro de 10 a 12 centímetros, a qual é chamada de bocha e dá nome ao jogo. $\mathrm{Na}$ tarefa em questão o "bolim" (bola de menor circunferência que é utilizada como alvo no jogo) foi trocado por um alvo circular com seis círculos que aumentavam em $10 \mathrm{~cm}$ de raio. Esses círculos foram pontuados de forma decrescente do círculo central (valor 6) ao mais externo (valor 1). O alvo ainda continha duas retas que se cruzavam formando um $\mathrm{X}$, e assim dividindo o alvo em quatro áreas triangulares, à frente, atrás, à esquerda e à direita e tinham o papel de informar a direção do erro. O conhecimento de resultado utilizado foi descritivo, sendo em magnitude e direção, respectivamente. Os sujeitos foram divididos aleatoriamente em três grupos $(n=12)$ : sem pós-conhecimento de resultado (SI), 5 segundos de pós-conhecimento de resultado (I5) e 10 segundos de intervalo pós-conhecimento de resultado (I10). Na fase de aquisição, os sujeitos praticaram 120 tentativas posicionados a 6,5 metros da extremidade do alvo e, após 3 minutos, foi realizado o Teste de Transferência 1, com 10 tentativas. Em seguida foi aplicado o Teste de Transferência 2, com o arremesso sendo realizado a 7,5 metros de distância da extremidade do alvo. O Teste de Transferência 3 foi aplicado após 10 minutos do término do Teste de Transferência 1, também com 10 tentativas. Não foi fornecido conhecimento de resultado nos testes. Na realização de cada arremesso, uma cortina posicionada a um metro do sujeito era abaixada assim que a bola a ultrapassava, impedindo a visão do resultado do arremesso.

O presente estudo foi submetido ao comitê de ética da Universidade Federal de Minas Gerais e aprovado sob o parecer numero 158/03.

\section{RESULTADOS}

Em relação à média do escore, os grupos experimentais apresentaram desempenho semelhante na fase de aquisição e nos testes (Tabela 1). Observou-se manutenção do desempenho no Teste de Transferência 1 e queda no desempenho nos Testes de Transferência 2 e 3.

Tabela 1: Média do escore e coeficiente de variação dos grupos experimentais, em blocos de 10 tentativas, para a fase de aquisição e testes.

\begin{tabular}{|c|c|c|c|c|c|c|}
\hline Grupos & \multicolumn{2}{|c|}{ SI } & \multicolumn{2}{|c|}{15} & \multicolumn{2}{|c|}{110} \\
\hline $\begin{array}{l}\text { Blocos de } \\
\text { tentativas }\end{array}$ & M & $C V$ & M & $C V$ & M & CV \\
\hline \multicolumn{7}{|c|}{ Aquisição } \\
\hline 1 & 1,2 & 1.75 & 1,1 & 1.45 & 1,4 & 1,36 \\
\hline 2 & 1,5 & 1.27 & 1,9 & 0.94 & 1,5 & 1,3 \\
\hline 3 & 1,8 & 1.2 & 1,2 & 1.5 & 1,6 & 1.3 \\
\hline 4 & $1, ?$ & 1.18 & 1,5 & 1.4 & 2 & 0.95 \\
\hline 5 & 1,4 & 1.43 & 1,8 & 1.1 & 2 & 1.05 \\
\hline 6 & 1,9 & 1.26 & 1,6 & 1.3 & 1,9 & 1.1 \\
\hline$?$ & 2,3 & 0.95 & 1,8 & 1,2 & 2,1 & 1.04 \\
\hline 8 & 2,1 & 1.04 & 1,7 & 1.1 & 2 & 1.1 \\
\hline 9 & $1, ?$ & 1.17 & 1,7 & 1.2 & $1, ?$ & 1.3 \\
\hline 10 & 1,9 & 1.1 & 2,2 & 1.1 & 2 & 1.1 \\
\hline 11 & 2,3 & 0.95 & 2,1 & 0.95 & 2 & 1.1 \\
\hline 12 & 1,9 & 1.1 & 1,6 & 1.25 & 2,1 & 1.04 \\
\hline \multicolumn{7}{|c|}{ Teste de Transferência 1} \\
\hline 1 & 1.23 & 2,1 & 1,8 & 1,23 & $1, ?$ & 1,23 \\
\hline \multicolumn{7}{|c|}{ Teste de Transferência 2} \\
\hline 1 & 1.5 & 1,8 & 1,06 & 1,3 & 1,3 & 1,38 \\
\hline \multicolumn{7}{|c|}{ Teste de Transferência 3} \\
\hline 1 & 1.86 & 1,3 & 1.1 & 1,37 & 1,4 & 1,36 \\
\hline
\end{tabular}

Uma ANOVA two-way (3 grupos x 12 blocos de tentativas) com medidas repetidas no último fator foi conduzida para a análise da fase de aquisição. Não foi encontrada diferença entre grupos $\left(\mathrm{F}_{2,33}=1,09\right.$, $\mathrm{p}=0,347)$ ou na interação entre grupos e blocos de tentativas $\left(\mathrm{F}_{22,363}=0,71, \mathrm{p}=0,834\right)$. No entanto, observou-se diferença entre blocos de tentativas $\left(\mathrm{F}_{11}\right.$, $\left.{ }_{22}=7,15, \mathrm{p}<0,001\right)$ e o teste de Tukey indicou diferenças significantes $(\mathrm{p}<0,05)$ do $1^{\circ}$ para o $6^{\circ}, 7^{\circ}, 8^{\circ}$, $10^{\circ}, 11^{\circ}$ e $12^{\circ}$ blocos de tentativas; do $2^{\circ}$ bloco para o $7^{\circ}, 10^{\circ}$ e $11^{\circ}$ blocos de tentativas; e do $3^{\circ}$ bloco para o $11^{\circ}$ bloco de tentativas. Outra ANOVA two- 
way (3 grupos $\mathrm{x} 4$ blocos) com medidas repetidas no último fator foi realizada para o último bloco da fase de aquisição e os testes. Os resultados não mostraram diferença no fator grupos $\left(\mathrm{F}_{2,33}=1,31\right.$, $\mathrm{p}=0,283$ ) e na interação entre grupos e blocos de tentativas $\left(\mathrm{F}_{6,99}=0,99, \mathrm{p}=0,432\right)$. Observou-se diferença entre blocos de tentativas $\left(\mathrm{F}_{3,6}=10,29\right.$, $\mathrm{p}=0,01)$. O teste de Tukey indicou queda do desempenho entre o último bloco de tentativas da fase de aquisição para os Testes de Transferência 2 $(\mathrm{p}<0,001)$ e Transferência $3(\mathrm{p}<0,001)$, e do Teste de Transferência 1 com os Testes de Transferência 2 $(\mathrm{p}<0,01)$ e Transferência $3(\mathrm{p}<0,01)$.

Foi utilizado o coeficiente de variação como medida de variabilidade. Uma ANOVA two-way (3 grupos $\mathrm{x}$ 12 blocos de tentativas) com medidas repetidas no último fator foi conduzida na fase de aquisição. Não foi encontrada diferença nos resultados no fator grupos $\left(F_{2,33}=1,34, p=0,27\right)$, entre blocos de tentativas $\left(\mathrm{F}_{11,22}=1,02, \mathrm{p}=0,46\right)$ e na interação entre grupos e blocos $\left(\mathrm{F}_{22,363}=1,48, \mathrm{p}=0,08\right)$. Uma outra ANOVA two-way (3 grupos $\mathrm{x} 4$ blocos de tentativas) com medidas repetidas no último fator foi realizada entre o último bloco da aquisição e os testes, a qual não encontrou diferença no fator grupos $\left(F_{2,33}=0,8\right.$, $\mathrm{p}=0,46)$, entre blocos de tentativas $\left(\mathrm{F}_{2,66}=0,07\right.$, $\mathrm{p}=0,93)$ e na interação entre grupos e blocos de tentativas $\left(\mathrm{F}_{4,66}=0,89, \mathrm{p}=0,48\right)$ (tabela 1$)$.

\section{DISCUSSÃO E CONCLUSÕES}

Os resultados encontrados corroboram as conclusões de Bilodeau e Bilodeau (3), Simmons e Snyder (11) e Godinho e Mendes (5), de que não houve efeito positivo do aumento do intervalo pós-conhecimento de resultado na aquisição de habilidades motoras. Todavia, o arremesso da bocha, como outras tarefas do mundo real, apresenta algumas particularidades. Dentre essas, o intervalo de tempo entre o término da execução do lançamento e o momento em que a bola pára. Esse tempo representa um intervalo anterior ao próprio pré-conhecimento de resultado. $\mathrm{O}$ tempo do intervalo pré-conhecimento de resultado foi controlado, todavia o tempo que a bola demorava a parar poderia também levar a um fortalecimento do feedback intrínseco, o que diminuiria a necessidade do intervalo pós-conhecimento de resultado. Esse fenômeno pode ter levado os grupos a desempenhos semelhantes (12). É importante considerar, ainda, que o desempenho parece ter sido influenciado por uma questão metodológica, relacionada à baixa precisão de informação das tentativas nulas. Quando a tentativa não recebia pontuação, não houve referência da distância em relação ao alvo, ou seja, o escore zero não permitia diferenciar um arremesso mais próximo de outro mais distante.

Becker (1) relaciona o aumento da complexidade da tarefa como um dos fatores para melhor evidenciar a influência do intervalo pós-conhecimento de resultado. No presente estudo, a complexidade da tarefa está sendo analisada ao comparar o arremesso da bocha com tarefas tradicionais de laboratório, que envolvem menor número de graus de liberdade. Mesmo o arremesso da bocha, sendo considerado uma tarefa mais complexa que as utilizadas tradicionalmente em laboratório, não foi suficiente para confirmar a sugestão de Becker (1). Em suma, a influência do intervalo pós-conhecimento de resultados na aquisição do arremesso da bocha não foi confirmada, o que leva a sugerir novos estudos com medidas mais precisas, de modo a aumentar a precisão da informação. Por exemplo, o erro absoluto medido em centímetros e a escolha de tarefas nas quais se possa isolar os intervalos de tempo mais claramente, como arremessos e chutes com características de habilidades balísticas, nos quais o objeto impulsionado chega rapidamente ao seu destino.

\section{CORRESPONDÊNCIA}

Márcio M. Vieira

Rua São João 772 A, Água Branca

32371-100 Contagem, Minas Gerais

BRASIL

marciogin@bol.com.br 


\section{REFERÊNCIAS}

1. Becker P.W. (2000). Are simple line-length estimation tasks productive for examining temporal locus of knowledge of results? Perceptual and Motor Skills 91: 801-802.

2. Becker P.W., Mussina C.M. \& Persons R.W. (1963). Intertrial interval delay of knowledge of results, and motor performance. Perceptual and Motor Skills 17: 559-563.

3. Bilodeau E.A. \& Bilodeau I.M. (1958) Variable frequency knowledge of results and the learning of a sample skill. Journal of Experimental Psychology 55: 379-383.

4. Gallagher J.D. \& Thomas J.R. (1980) Effects of varying post-KR intervals upon children's motor performance. Journal of Motor Behavior 12: 41-56.

5. Godinho M. \& Mendes R. (1996) Aprendizagem Motora: informação de retorno sobre o resultado. Lisboa: Edições FMH.

6. Magill R.A. (1988) Activity during the post-knowledge of result interval can benefit motor skill learning. In O.G. Meijer \& K. Roth (Eds.) Complex Motor Behavior: 'The' motor-action controversy. Amsterdam: Elsevier, 231- 246.

7. Magill R.A. (1994) The influence of augmented feedback on skill learning depends on characteristics of the skill and the learner. Quest 46: 314-327.

8. Ramella R.J. (1983) Processing, knowledge of results, and a multi-dimensional task. Perceptual and Motor Skills 57: 4348.

9. Salmoni A.W., Schimdt R.A. \& Walter C.B. (1984) Knowledge of results and motor learning: a review and critical reappraisal. Psychological Bulletin 95: 355-386.

10. Schimdt R.A. (1988) Motor control and learning: a behavioral emphasys. ( $2^{\text {nd }}$ ed.) Champaign, Il: Human Kinetics.

11. Simmons R.W. \& Snyder R.J. (1983) Variation of temporal locus of knowledge of results: effects on motor performance of a simple task. Perceptual and Motor Skills 56: 399-404.

12. Swinnen S.P., Schimdt R.A., Nicholson D.E. \& Shapiro D.C. (1990). Information feedback for skill acquisition: instantaneous knowledge results degrades learning. Journal of Experimental Psychology: Learning, Memory and Cognition 16: 706-716.

13. Travlos A.K. (1999) Re-examining the temporal locus of knowledge of results (KR): A self-paced approach to learning. Perceptual and Motor Skills 89: 1073-1087.

14. Travlos A.K. \& Pratt J. (1995). The temporal locus of knowledge of results: a meta-analytic review. Perceptual and Motor Skills 80:3-14.

15. Weinberg D.R., Guy D.E. \& Tupper R.W. (1964). Variations of post feedback interval in simple motor learning. Journal of Experimental Psychology 67: 98-99. 